\title{
The garden of antimicrobial delights
}

\section{Julian Davies}

\author{
Address: Department of Microbiology and Immunology, Life Science Institute, 2350 Health Sciences Mall, University of British Columbia, \\ Vancouver, BC V6T 1Z3, Canada \\ Email: jed@interchange.ubc.ca
}

Fl000 Biology Reports 2010, 2:26 (doi:10.34I0/B2-26)

The electronic version of this article is the complete one and can be found at: http://fl000.com/reports/biology/content/2/26

\begin{abstract}
Major advances in genomics, cloning and chemistry will re-stock the dwindling supply of effective antimicrobials and meet the threat of antibiotic resistance development.
\end{abstract}

\section{Introduction and context}

Almost all papers and reviews dealing with infectious diseases stress that new classes of antibiotics are badly needed to treat the increasing number of common and emerging cases of infection due to antibiotic-resistant organisms, be they bacteria, viruses or parasites. What happened to the optimism of the late 1960s when the then Surgeon General of the US announced 'we can close the book on infectious diseases'? For some years now it has been clear that the situation with respect to the treatment of common bacterial infections, especially in hospitals, is poor, if not dire [1]. Physicians have a diminishing armamentarium of antibiotics available for clinical use; most of the older compounds are ineffective against present-day resistant strains and reinforcements are few and far between. Out-patient infections may still be treatable with old standbys such as ampicillin and related derivatives; however, in many of these cases the infectious organism is not identified and the cure may have been more a result of natural recovery of the patient. The few novel compounds available are reserved for lastresort use and only in critical care units of hospitals.

If we are to continue with the current practices in the treatment of infectious disease, new and reliable sources of novel antimicrobials are badly needed for all types of microbial diseases. Where will they come from? And who will produce them? Much has been written by, and about, the pharmaceutical industry focusing on the difficulties of finding effective bioactive compounds; this is coupled with comments on the poor profit return on research in this area. Not surprisingly, the industry is strongly disinclined to continue their efforts. I shall not go into details of this pathetic 'histoire', enough has been written on it by others closer to the problem [2]. However, in this commentary I will take issue with the oft-repeated assertion that it is 'hard' to find new compounds with the required therapeutic activity from nature, the assumption being that Big Pharma has exhaustively screened all of the available sources of bioactive compounds and that slim pickings remain. Emerging technological advances make it increasingly clear that this stance is indefensible, as described below.

\section{Major recent advances}

If we assume that upwards of 100,000 different strains of bacteria and fungi may have been screened for therapeutic agents by the industry [3], this represents but a fraction of the population of the microbial kingdoms (a gram of soil may contain a thousand different bacterial species). There are likely billions of antibiotic-producing strains in the biosphere, each with the potential to produce 10 times this number in terms of bioactive small molecules with drug potential. This information comes from two sources: firstly, more than $90 \%$ of microbes cannot be grown under laboratory conditions; this includes all possible environmental sources: marine, terrestrial, endosymbionts, and so on. Secondly, extensive genome sequencing over the past 10 years has revealed that one of the most productive bacterial sources of antibiotics and other therapeutic agents, the streptomycetes, possesses the genetic capacity to make as 
many as 30 different bioactive small molecules [4]. The so-called cryptic biosynthetic pathways are not expressed (or are poorly expressed) under traditional fermentation conditions, and their products have been missed because of inadequate and insensitive screening processes. It is safe to say that with increasing genomic analyses the potential for the discovery of many new structural entities will be enhanced considerably [5].

This treasure trove of bioactive compounds will not yield its secrets and benefits easily. New methodology must be developed to grow fastidious microbes in the laboratory and creative approaches to the cloning and expression of identified, but product-unknown, biosynthetic pathways must be developed; new expression hosts and pathway expression processes are essential for success. Another major hurdle is the isolation, purification and structure determination of the compounds produced by the microbial hosts; traditional natural product chemistry is almost a dark art, with very few skilled practitioners worldwide. However, new methods of cell-based screening, compound isolation and structure determination are coming on the scene; increasingly sensitive instrumentation may resolve other aspects of the discovery problem. New types of high-throughput automated isolation procedures with integrated spectroscopic approaches to molecular analysis are becoming available and these should eventually simplify and solve the issue of rapid identification of novel chemistry [6]. At first these approaches will be expensive, of course, but the need is there and if the spirit is willing, with continual technical advances, complementary analytical approaches (that may be more affordable and user-friendly) will become available. There is an excellent model to be found from the revolution in DNA sequencing; it took several billions of dollars to obtain a draft nucleotide sequence of the human genome in 2000, 10 years later we are speaking of $\$ 1000$ genomes (or less!) [7]. More importantly, for low molecular weight bioactives, microbial genome sequences will soon be priced at $\$ 10$ a shot and completed in a day. Antibiotic discovery will start with the complete nucleotide sequence of a biosynthetic pathway for an unknown compound, a complete reverse of the traditional approach!

Of course, the identified pathways still have to be converted into products, but prototypes for this type of engineering are already established; even Escherichia coli is considered a good candidate as expression host [8]. In addition, the possibility of creating new types of molecules by making hybrid biosynthetic pathways is a field of investigation that will develop in parallel with these approaches [9]. Engineering the amino acid components of non-ribosomal peptides is yet another method that shows promise for the production of nonnatural molecules in vivo [10].

To capitalize on the increasing availability of new classes of small-molecule therapeutics, comparable advances in high-throughput screening and testing of their diverse bioactivities, using cell-based assays, will be essential, and one can predict more extensive use of reliable insect and worm models of human disease [11]. Imagine screening thousands of microbial compounds against hundreds of disease syndromes in microtiter plates! Many of the necessary procedures are already in place but they need to be made amenable to high-throughput analysis. There is a huge world of bioactive small molecules (the Parvome) waiting to be exploited for all aspects of therapeutics!

An additional factor for success in antibiotic discovery in the near future will come from a more comprehensive understanding of natural microbial communities and their interactions with their hosts, especially in humans; our health is critically dependent on gut microbiota. The rapidly increasing knowledge of this type of dependency, when used in a predictive sense, will surely identify more drug targets and more useful drug leads. Rapidly advancing studies of the nature of human and animal microbiomes are revealing extensive roles of commensal microbes in the health and maintenance of their complex hosts. Advances in this field will surely reveal unanticipated drug targets for a variety of afflictions; can we envisage a new branch of industry 'Micro Pharma'?

\section{Future directions}

All of the above technological advances (and more, such as systems biology and metabolic network mapping) will contribute to the means of producing newer generations of antimicrobial agents. However, it is time to come back down to earth. No matter what is done to revolutionise the drug industry, it is necessary for the dispensers and users of antibiotics to realize that bacteria are immortal; all treatments should be carefully controlled so that we can avoid or reduce the same problems with antibiotic resistance development that have made so many good drugs redundant in the recent past. And to finish with stark reality, although the US Food and Drug Administration rules, perhaps they also can mutate with new solutions for health care needs.

To conclude, we must consider who will be responsible for the implementation of these revolutionary developments in the process of drug discovery and development? We cannot wait for the biopharmaceutical industry to do this. Big Pharma is too hidebound by the tradition of 
over-management, and 'small' biotech is too dependent on venture capital (a misnomer if ever there was one; where is the adventure in modern venture capital?). What is needed is a new drug discovery model; perhaps university research combined with government grant funding could lead to ways of enhancing the development of early-stage discovery in academia. A number of universities around the world have initiated pre-clinical, pre-industry discovery groups with the goal of bridging the freedom and originality of academic research with the hard facts of drug development. This should be the way of the future!

\section{Competing interests}

The author declares that he has no competing interests.

\section{Acknowledgments}

Work in the author's laboratory has been supported by the National Science and Engineering Council, the Canadian Institutes of Health and the Tally Fund.

\section{References}

I. Spellberg B, Guidos R, Gilbert D, Bradley J, Boucher HW, Scheld WM, Bartlett JG, Edwards J Jr; Infectious Diseases Society of America: The epidemic of antibiotic-resistant infections: a call to action for the medical community from the Infectious Diseases Society of America. Clin Infect Dis 2008, 46:155-64.

FI000 Factor 6.0 Must Read

Evaluated by Antonio Cassone 15 Jan 2008
2. Payne $D$, Tomasz $A$ : The challenge of antibiotic resistant bacterial pathogens: the medical need, the market and prospects for new antimicrobial agents. Curr Opin Microbiol 2004, 7:435-8.

3. Baltz RH: Marcel Faber Roundtable: is our antibiotic pipeline unproductive because of starvation, constipation or lack of inspiration? J Ind Microbiol Biotechnol 2006, 33:507-I3.

4. Ohnishi $Y$, Ishikawa J, Hara $H$, Suzuki $H$, Ikenoya $M$, Ikeda $H$, Yamashita A, Hattori M, Horinouchi S: Genome sequence of the Streptomycin-producing microorganism Streptomyces griseus IFO I3350. J Bacteriol 2008, 190:4050-60.

5. Zerikly M, Challis GL: Strategies for the discovery of new natural products by genome mining. Chembiochem 2009, 10:625-33.

6. Esquenazi E, Yang YL, Watrous J, Gerwick WH, Dorrestein PC: Imaging mass spectrometry of natural products. Nat Prod Rep 2009, 26: $152 \mid-34$.

7. Service RF: Gene sequencing: the race for the $\$ 1000$ genome. Science 2006, 3 I I: I544-6.

8. Pfeifer B, Hu Z, Licari P, Khosla C: Process and metabolic strategies for improved production of Escherichia coli-derived 6-deoxyerythronolide B. Appl Envir Microbiol 2002, 68:3287-92.

9. Sheehan LS, Lill RE, Wilkinson B, Sheridan RM, Vousden WA, Kaja AL, Crouse GD, Gifford J, Graupner PR, Karr L, Lewer P, Sparks TC, Leadlay PF, Waldron C, Martin CJ: Engineering of the spinosyn PKS: directing starter unit incorporation. J Nat Prod 2006, 69: $1702-10$.

10. Doekel S, Coëffet-Le Gal M-F, Gu J-Q, Chu M, Baltz RH, Brian P: Non-ribosomal peptide synthetase module fusions to produce derivatives of daptomycin in Streptomyces roseosporus. Microbiology 2008, I54:2872-80.

II. Moy TI, Conery AL, Larkins-Ford J, Wu G, Mazitschek R, Casadei G, Lewis K, Carpenter AE, Ausubel FM: High-throughput screen for novel antimicrobials using a whole animal infection model. ACS Chem Biol 2009, 4:527-33.

FI000 Factor 6.0 Must Read

Evaluated by David Triggle 25 Nov 2009 